Pacific Journal of Mathematic

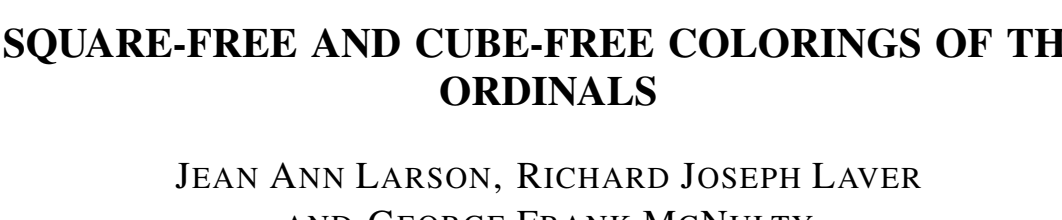




\section{SQUARE-FREE AND CUBE-FREE COLORINGS OF THE ORDINALS}

Jean A. Larson, Richard Laver and George F. MCNulty

We prove: Theorem 1 . The class of all ordinals has a square-free 3-coloring and a cube-free 2-coloring. Theorem 2 . Every $k$ th power-free $n$-coloring of $\alpha$ can be extended to a maximal $k$ th power-free $n$-coloring of $\beta$, for some $\beta \times \alpha \cdot \omega$, where $k, n \in \omega$.

Every ordinal is conceived as the set of all smaller ordinals; $\omega$ is the least infinite ordinal. By an interval of ordinals we mean any set $\{\delta: \beta \leqq \delta<\gamma\}$ where $\beta$ and $\gamma$ are ordinals; $[\beta, \gamma)$ abbreviates $\{\delta: \beta \leqq \delta<\gamma\}$. If $S$ and $T$ are intervals then there can be at most one order isomorphism from $S$ onto $T$.

Let $S$ be an interval of ordinals and $\kappa$ be a cardinal. A $\kappa$-coloring of $S$ is just a function with domain $S$ and range included in $\kappa$. Suppose $S$ and $T$ are intervals of ordinals and that $f$ is a coloring of $S$ while $g$ is a coloring of $T$. Then the coloring $f$ of $S$ is similar to the coloring $g$ of $T$ provided $S$ and $T$ are order isomorphic and $f(\alpha)=g(h(\alpha))$ for all $\alpha \in S$ where $h$ is the unique order isomorphism from $S$ onto $T$; if $f$ and $g$ are clear from the context we say that $S$ is similar to $T$. A coloring $f$ of the ordinal $\alpha$ is square-free if no two adjacent nonempty intervals of $\alpha$ are similar; it is cube-free if no three consecutive nonempty intervals are all similar to each other. All these notions extend naturally to the class of all ordinals.

In Bean, Ehrenfeucht, and McNulty [1] it was shown that $\alpha$ has a square-free 3-coloring and a cube-free 2-coloring whenever $\alpha<\left(2^{\aleph_{0}}\right)^{+}$and the question of extending this result to all ordinals was left open. This question is resolved here.

THEOREM 1. The class of all ordinals has a square-free 3-coloring and a cube-free 2-coloring.

If $I$ is a class of ordinals and $\alpha_{\beta}$ is an ordinal for each $\beta \in I$, then $\sum_{\beta \in I} \alpha_{\beta}$ denotes the ordinal sum of the $\alpha_{\beta}$ 's with respect to I. (See Sierpinski [2] for details.) Finite ordinal sums are written like $\alpha_{0}+\alpha_{1}+\cdots+\alpha_{n-1}$. For each $\beta \in I$, let $\operatorname{Int}(\beta)=\left[\mu, \mu+\alpha_{\beta}\right)$ where $\mu=\sum_{\gamma \in J} \alpha_{\gamma}$ and $J=I \cap \beta$. For each $\beta \in I$, Int $(\beta)$ is order isomorphic with $\alpha_{\beta}$. In fact, $\sum_{\beta \in I} \alpha_{\beta}$ can be construed as the disjoint union of the $\operatorname{Int}(\beta)$ 's as $\beta \in I$ where the intervals are given the order type of $I$. This means that if $f_{\beta}$ is a $\kappa$-coloring of $\alpha_{\beta}$, 
for each $\beta \in I$, then there is a $\kappa$-coloring $f$ of $\sum_{\beta \in I} \alpha_{\beta}$ such that $f \uparrow \operatorname{Int}(\beta)$ is similar to $f_{\beta}$.

An ordinal $\alpha$ is (additively) indecomposable provided $\alpha \neq \beta+\gamma$ whenever $\beta<\alpha$ and $\gamma<\alpha$. It is known (cf. Sierpinski [2]) that every ordinal is the ordinal sum of finitely many indecomposable ordinals and that the infinite indecomposable ordinals are exactly the ordinal powers of $\omega$.

LEMMA 0. If $\alpha$ is the class of all ordinals or $\alpha$ is an indecomposable ordinal with $\alpha>\omega$, then $\alpha$ is the sum of a strictly increasing sequence of smaller limit ordinals.

Proof. There are three cases. First, suppose $\alpha=\omega^{\beta}$ where $\beta$ is a limit ordinal. So $\alpha=\omega^{\beta}=\sum_{\gamma<\beta} \omega^{\gamma}$. Second, suppose $\alpha=\omega^{\beta+1}$. Then $\alpha=\omega^{\beta+1}=\omega^{\beta} \cdot \omega=\sum_{n \in \omega}\left(\omega^{\beta} \cdot n\right)$. Third, the class of all ordinals is $\sum_{\kappa \in I} \kappa$, where $I$ is the class of cardinals. In each case the lemma holds.

Let $f$ be a coloring of the interval $S$ of ordinals and let $g$ be a coloring of the interval $T . S$ and $T$ are mismatched provided that $U$ and $V$ fail to be similar whenever $U$ is an infinite subinterval of $S$ and $V$ is an infinite subinterval of $T$. Theorems 1.8 and 1.16 from Bean, Ehrenfeucht, and McNulty [1] are collected in the next lemma.

LEMMA 1. (a) There is a collection $\mathscr{F}$ of square-free 3-colorings of $\omega$ such that $|\mathscr{F}|=2^{\aleph_{0}}$ and $C$ and $D$ are mismatched whenever $C, D \in \mathscr{F}$ with $C \neq D$.

(b) There is a collection $\mathscr{S}$ of cube-free 2-colorings of $\omega$ such that $|\mathscr{S}|=2^{\aleph_{0}}$ and $C$ and $D$ are mismatched whenever $C, D \in \mathscr{S}$ with $C \neq D$.

Proof of Theorem 1. We will provide a proof that the class of all ordinals has a square-free 3-coloring. This proof can be easily modified to establish that the class of all ordinals has a cube-free 2-coloring. The property of having a square-free 3-coloring is hereditary in the sense that if $\alpha$ has a square-free 3-coloring and $\beta<\alpha$, then $\beta$ has a square-free 3-coloring. Below we are concerned with providing each limit ordinal with a square-free 3-coloring and we proceed by induction.

Induction hypothesis. If $\alpha$ is an infinite limit ordinal or the class of all ordinals, and $f_{0}, f_{1}, \cdots$ are countably many square-free 3 -colorings of $\omega$ such that $f_{i}$ and $f_{j}$ are mismatched whenever $i, j \in \omega$ with $i \neq j$, then there is a 3-coloring $g$ of $\alpha$ such that 
(i) $g$ is square-free.

(ii) $g$ and $f_{i}$ are mismatched for each $i \in \omega$.

(iii) Any two similar infinite intervals of $\alpha$ are separated by an infinite interval.

Suppose the induction hypothesis holds for all infinite limit ordinals less than $\alpha$ and that $f_{0}, f_{1}, f_{2}, \cdots$ are countably many pairwise mismatched square-free 3-colorings of $\omega$. There are two cases.

Case 1. $\alpha=\rho_{0}+\rho_{1}+\cdots+\rho_{n}$ where $\rho_{0}, \cdots, \rho_{n}$ are indecomposable and $0<n \in \omega$.

According to Lemma 1 there must be $h_{0}, \cdots, h_{n}$, all square-free 3-colorings of $\omega$, such that $h_{0}, h_{1}, \cdots, h_{n}, f_{0}, f_{1}, \cdots$ are all pairwise mismatched. By the induction hypothesis there are 3-colorings $d_{0}, \cdots, d_{n}$ of $\rho_{0}, \cdots, \rho_{n}$ respectively such that for each $i \leqq n$

(i $)^{\prime} \quad d_{i}$ is square-free.

(ii) $d_{i}, h_{0}, h_{1}, \cdots, h_{n}, f_{0}, f_{1} \cdots$ are all pairwise mismatched.

(iii)' Any two similar infinite intervals of $\rho_{i}$ are separated by an infinite interval.

For each $i \leqq n$ and each $\gamma \in \rho_{i}$, let

$$
d_{i}^{*}(\gamma)= \begin{cases}h_{i}(\gamma) & \text { if } \gamma \in \omega \\ d_{i}(\gamma) & \text { otherwise }\end{cases}
$$

and let $g$ be the coloring of $\alpha$ induced by $d_{0}^{*}, \cdots, d_{n}^{*}$.

Condition (ii) of the induction hypothesis holds by (ii)'. To check condition (iii) suppose $S$ and $T$ are distinct similar infinite intervals of $\alpha$. Since $h_{i}$ and $d_{j}$ are mismatched whenever $i, j \leqq n$ and since $h_{0}, h_{1}, \cdots, h_{n}$ are pairwise mismatched, for each $i \leqq n$ there is exactly one interval $U$ of $\alpha$ (of order type $\omega$ ) such that $f \mid U$ is similar to $h_{i}$. Since $S$ and $T$ are distinct but similar neither can have a subinterval similar to any of $h_{0}, h_{1}, \cdots, h_{n}$ or any of their final segments. Consequently there are $i, j \leqq n$, with finite initial segments $\delta$ of $\rho_{i+1}$ and $\varepsilon$ of $\rho_{j+1}$ such that $S$ is a subinterval of $\rho_{i}+\delta$ missing the initial segment of $\rho_{i}$ of order-type $\omega$, while $T$ is a subinterval of $\rho_{j}+\varepsilon$ missing the initial segment of $\rho_{j}$ of order-type $\omega$. If $i \neq j$ then (iii) follows immediately, so suppose $i=j$. There must be cofinite initial segments $S^{\prime}$ of $S$ and $T^{\prime \prime}$ of $T$ such that $S^{\prime}$ and $T^{\prime}$ are distinct yet similar and both $S^{\prime}$ and $T^{\prime}$ are subintervals of $\rho_{i}$ missing the initial segment of $\rho_{i}$ of order type $\omega$. So $S^{\prime}$ and $T^{\prime}$ are colored by $d_{i}$ and by (iii) they are separated by an infinite interval and therefore $S$ and $T$ are separated by an infinite interval as well.

To see that $g$ is a square-free coloring of $\alpha$, observe that (iii) forces any two similar adjacent intervals to be finite. But $g$ was 
devised so that all intervals of $\alpha$ of order type $\omega$ are colored in a square-free manner. Hence $g$ is square-free and Case I of the induction is complete.

Case II. $\alpha$ is indecomposable with $\alpha>\omega$.

By Lemma $0 \alpha=\sum_{r \in \beta} \rho_{\gamma}$ for some $\beta$ where $\rho_{\gamma}<\rho_{\dot{s}}<\alpha$ and $\rho_{i}$ is an infinite limit ordinal, if $\gamma<\delta<\beta$. According to Lemma 1 there must be $h_{0}$ and $h_{1}$, both square-free 3-colorings of $\omega$ such that $h_{0}, h_{1}, f_{0}, f_{1}, f_{2}, \cdots$ are pairwise mismatched. By the induction hypothesis for each $\gamma \in \beta$ there is a 3-coloring $d_{r}$ of $\rho_{r}$ such that

(i $)^{\prime \prime} \quad d_{r}$ is square-free.

(i!)" $d_{\gamma}, h_{0}, h_{1}, f_{0}, f_{1}, f_{2}, \cdots$ are pairwise mismatched.

(iii)" Any two similar infinite intervals of $\rho_{i}$ are separated by an infinite interval

$$
d_{r}^{*}(\delta)= \begin{cases}h_{0}(\delta) & \text { if } \delta \in \omega \text { and } \gamma \text { is even } \\ h_{1}(\delta) & \text { if } \delta \in \omega \text { and } \gamma \text { is odd } \\ d_{i}(\delta) & \text { otherwise }\end{cases}
$$

and let $g$ be the coloring of $\alpha$ induced by $\left\langle d_{\gamma}^{*}: \gamma \in \beta\right\rangle$.

Conditions (i) and (ii) of the induction hypothesis can be established as in Case I. We argue that (iii) holds. Suppose $S$ and $T$ are similar infinite intervals in $\alpha$. If $S$ contains an interval of type $\omega$ colored the way $h_{0}\left(\right.$ or $h_{1}$ ) colors some final segment of $\omega$ then the same is true for $T$. According to the construction of $g$ these kinds of colorings occur only on the initial segments of each $\rho_{r}$ of type $\omega$. Since the $\rho_{r}$ 's form an increasing sequence, no interval between an interval colored with $h_{0}$ and the next colored with $h_{1}$ occurs twice. So if $S$ contains an interval of type $\omega$ colored the way $h_{0}$ (or $h_{1}$ ) colors some final segment of $\omega$, then $S$ does not contain an interval of type $\omega$ colored the way $h_{1}$ (alternatively $h_{0}$ ) colors some final segment of $\omega$. The same is true for $T$. Consequently, if $S$ and $T$ were separated by a finite interval, then both $S$ and $T$ would lie entirely in $\rho_{\gamma}+\delta$ for some $\gamma$ where $\delta$ is a finite initial segment of $\rho_{r_{+1}}$. From this point the argument proceeds as in Case I.

Since Lemma 1 guarantees the theorem when $\alpha=\omega$, the induction is complete and the theorem established.

For any $k \in \omega$, kth power-free colorings have definitions analogous to those of square-free and cube-free colorings. Every squarefree coloring is $k$ th power-free for all $k \geqq 2$. A $k$ th power-free $\kappa$-coloring $f$ of $\alpha$ is maximal provided $f$ cannot be extended to a $k$ th power-free $\kappa$-coloring of $\alpha+1$. In Bean, Ehrenfeucht, and McNulty [1] it is shown that every $k$ th power-free $n$-coloring $f$ of $m$ can be 
extended to a maximal $k$ th power-free $n$-coloring of some natural number, whenever $k, n, m \in \omega$. We remark that the following theorem holds. The proof differs in no important way from the proof of Theorem 2.0 in [1].

THEOREM 2. For any natural numbers $n$ and $k$ and any ordinal $\alpha$, every kth power-free n-coloring of $\alpha$ can be extended to a maximal kth power-free n-coloring of $\beta$ for some $\beta \in \alpha \cdot \omega$.

\section{References}

1. D. Bean, A. Ehrenfeucht, and G. McNulty, Avoidable patterns in strings of symbols, Pacific J. Math., 85 (1979), 261-294.

2. V. Sierpinski, Cardinal and Ordinal Numbers, Warsawa 1958.

Received February 7, 1978.

UNIVERSITY OF FLORIDA

GAINESVILLE, FL 32611

UNIVERSITY OF COLORADO

BOULDER, CO 80302

AND

University of South Carolina

Columbia SC 29208 



\section{PACIFIC JOURNAL OF MATHEMATICS}

\section{EDITORS}

DONALD BABBITT (Managing Editor)

University of Galifornia

Los Angeles, California 90024

Hugo RossI

University of Utah

Salt Lake City, UT 84112

C. C. MoOre AND ANDrew OGG

University of California

Berkeley, CA 94720

\section{J. DugundjI}

Department of Mathematics University of Southern California

Los Angeles, California 90007

R. Finn and J. Milgram

Stanford University

Stanford, California 94305

ASSOCIATE EDITORS

E. F. Beckenbachi

B. H. NeumanN

F. WOLF

K. YOSHIDA

\section{SUPPORTING INSTITUTIONS}

UNIVERSITY OF BRITISH COLUMBIA

CALIFORNIA INSTITUTE OF TECHNOLOGY

UNIVERSITY OF CALIFORNIA

MONTANA STATE UNIVERSITY

UNIVERSITY OF NEVADA, RENO

NEW MEXICO STATE UNIVERSITY

OREGON STATE UNIVERSITY

UNIVERSITY OF OREGON
UNIVERSITY OF SOUTHERN CALIFONIA

STANFORD UNIVERSITY

UNIVERSITY OF HAWAII

UNIVERSITY OF TOKYO

UNIVERSITY OF UTAH

WASHINGTON STATE UNIVERSITY

UNIVERSITY OF WASHINGTON

Printed in Japan by International Academic Printing Co., Ltd., Tokyo, Japan 


\section{Pacific Journal of Mathematics \\ Vol. 89 , No. 1 \\ May, 1980}

David Bressoud, A note on gap-frequency partitions ................ 1

John David Brillhart, A double inversion formula ................. 7

Frank Richard Deutsch, Günther Nürnberger and Ivan Singer, Weak

Chebyshev subspaces and alternation .......................

Edward Richard Fadell, The relationship between Ljusternik-Schnirelman

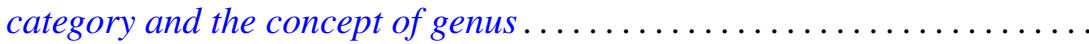

Harriet Jane Fell, On the zeros of convex combinations of polynomials.......

John Albert Fridy, An addendum to: "Tauberian theorems via block

dominated matrices" ..................................

Andrzej Granas, Ronald Bernard Guenther and John Walter Lee, Applications of topological transversality to differential equations. I. Some nonlinear

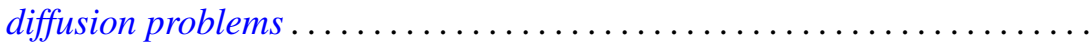

David E. Handelman and G. Renault, Actions of finite groups on self-injective

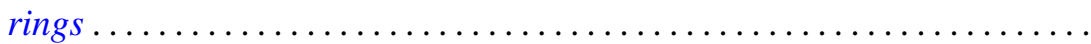

Michael Frank Hutchinson, Local $\Lambda$ sets for profinite groups .............

Arnold Samuel Kas, On the handlebody decomposition associated to a

Lefschetz fibration...

Hans Keller, On the lattice of all closed subspaces of a Hermitian space.....

P. S. Kenderov, Dense strong continuity of pointwise continuous

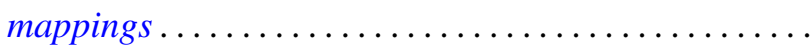

Robert Edward Kennedy, Krull rings.................

Jean Ann Larson, Richard Joseph Laver and George Frank McNulty,

Square-free and cube-free colorings of the ordinals ...

Viktor Losert and Harald Rindler, Cyclic vectors for $L^{p}(G)$

John Rowlay Martin and Edward D. Tymchatyn, Fixed point sets of

1-dimensional Peano continua...

Augusto Nobile, On equisingular families of isolated singularities ...

Kenneth Joseph Prevot, Imbedding smooth involutions in trivial bundles ...

Thomas Munro Price, Spanning surfaces for projective planes in four space.

Dave Riffelmacher, Sweedler's two-cocycles and Hochschild cohomology....

Niels Schwartz, Archimedean lattice-ordered fields that are algebraic over

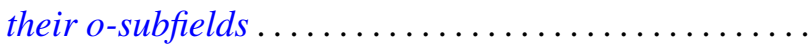

Chao-Liang Shen, A note on the automorphism groups of simple dimension groups.

Kenneth Barry Stolarsky, Mapping properties, growth, and uniqueness of

Vieta (infinite cosine) products ...

Warren James Wong, Maps on simple algebras preserving zero products. I.

The associative case ............................. 